\title{
Nanosheet-Structured Boron Carbon Nitride Spheres as Stable Electrocatalyst Support for Oxygen Reduction Reaction
}

\author{
Fei Liu ${ }^{1}{ }^{2 *}$, Yaqi Ren ${ }^{1}$, Xixi Ji ${ }^{1}$ \\ ${ }^{1}$ Department of Material Science and Engineering, Shenzhen Graduate School, Harbin Institute of \\ Technology, 518055, China. \\ ${ }^{2}$ School of Environmetal Science \& Engineering, Southern University of Science and Technology, 518055, \\ China.
}

* Corresponding author. Tel.: +8613510182230; email: liuf@sustc.edu.cn

Manuscript submitted April 5, 2017; accepted September 15, 2017.

doi: 10.17706/ijmse.2017.5.4.123-132

\begin{abstract}
A novel nanosheet-structured boron carbon nitride spheres (BCNSs) have been prepared for application as electrocatalyst support of oxygen reduction reaction (ORR) for the first time. The BCNSs were prepared by a catalyzed thermal evaporation method from solid B powders. The BCNSs are hollow with the diameter in the range of 450-500 $\mathrm{nm}$ and composed of many radially oriented ultrathin nanosheets with a mean thickness of $8.6 \mathrm{~nm}$. Due to immobilization in the spheres the constituting BCN nanosheets are free of agglomeration, capable of preserving high surface area during application. Small Pt nanoparticles with diameters of 2-8 $\mathrm{nm}$ were deposited onto the BCNSs by ethylene glycol reduction method. The Pt/BCNSs catalyst shows excellent electrocatalytic performance for ORR with the onset potential at $0.10 \mathrm{~V}$ and current retention of $85.4 \%$ after 20000 s, which is superior to the commercial $20 \% \mathrm{Pt} / \mathrm{C}$ catalyst. The improved electrocatalytic performance of the Pt/BCNSs arises from the unique structure and high chemical stability of the BCNSs support and the possible synergistic effect between Pt and the support. The present work indicates that the BCNSs are excellent electrocatalyst support for ORR, which can be further improved by optimizing the composition and structure.
\end{abstract}

Key words: Boron carbon nitride, nanosheets, spheres, oxygen reduction reaction.

\section{Introduction}

Fuel cell is regarded as one of the most promising energy conversion devices due to its high energy conversion efficiency [1], low emission [2], and high energy density [3]. The cathode electrocatalysts for oxygen reduction reaction (ORR) play a key role in the running of the fuel cells [4], [5]. Platinum (Pt) is conventionally used as the electrocatalysts [6]-[8]. Generally, the Pt catalysts are used by dispersing on various supports, which is necessary for obtaining excellent electrocatalytic performance [9], [10]. Carbon black has been widely used as the electrocatalyst support due to the high specific surface area (SSA) and good electrical conductivity [11]. However, the carbon black in the form of particles suffers from the problems of agglomeration and electrochemical corrosion, resulting in the performance decrease of the catalysts [12], [13]. Recently, many research groups have studied alternative non-carbonaceous supports of electrocatalysts such as carbides [14], nitrides [15], and transition metal oxides [16], [17]. Although these materials appear promising for making high efficient electrocatalytic systems of ORR, some obstacle issues 
such as small specific surface area or poor electrical conductivity still degrade the performance of the supported catalysts [18]-[20]. Therefore, it is necessary to develop novel electrocatalyst supports and thus maximize the performance of the electrocatalysts.

Hexagonal boron nitride (BN) with the graphite-like structure has been used as the catalyst supports for chemical engineering, which shows excellent performance due to the high chemical and thermal stability [21]-[23]. However, the poor electrical conductivity of BN constitutes a big obstacle for its application as electrocatalyst support for ORR. Boron carbon nitride (BCN) formed by introducing carbon atoms into the BN structure possesses much improved electrical conductivity comparing with BN and much improved chemical and thermal stability comparing with carbon [24], [25], which endows BCN the superiority for application as the electrocatalyst support. Recent reports indicate that the BCN materials possess electrocatalytic activity for ORR [26]-[28]. However, to the best of our knowledge, the application of the BCN materials as electrocatalyst support to immobilize Pt nanoparticles for ORR has not been reported yet so far.

Appropriate nanostructure with high effective surface area and abundant active adsorption sites is required for the catalyst supports to generate excellent catalytic performance. Different nanostructures such as nanowires [29] and graphene [30], [31] have been used to support Pt catalyst for fuel cell application. As a typical two-dimensional (2D) nanomaterial, BCN nanosheets may be a good candidate of Pt catalyst support due to its higher surface area and abundant edge sites with high activity [24]. However, the 2D nanosheets generally show a strong tendency to agglomerate [32], which leads to great loss of the effective surface area and thus the catalytic performance. Recently, by a catalyzed thermal evaporation process we prepared nanosheet-structured BN spheres [32], in which the BN nanosheets are radially oriented with the sheet edges oriented on the surface. For this architecture, the BN nanosheets are fixed and the problem of agglomeration is thus avoided. Due to full exposure of the nanosheet surface and presence of highly active edge sites on the sphere surface, the nanosheet-structured BN spheres exhibit excellent adsorption performance for many molecules and ions. It is expected that the nanosheet-structured BCN spheres (BCNSs) with similar structure may be good Pt supports for ORR. However, the BCNSs with radially grown nanosheets have not been prepared yet.

In this paper, we prepared BCNSs by a simple catalyzed thermal evaporation approach and investigated their application as Pt catalyst support for ORR. The BCNSs are composed of radially grown ultrathin BCN nanosheets with the sheet edges oriented on the surface [32]. Due to the superior structure of no agglomeration and radially orientation of the nanosheets and high chemical stability of the BCN material, the Pt/BCNSs catalyst shows excellent electrocatalytic performance for ORR with onset potential as high as $0.10 \mathrm{~V}$ and stability up to $85.4 \%$ current retention after $20000 \mathrm{~s}$.

\section{Experimental}

The BCNSs were prepared by a catalyzed thermal evaporation method from solid B powders. A Mixture of $\mathrm{CoFe}_{2} \mathrm{O}_{4}$ and $\mathrm{CoO}$ with a molar ratio of $1: 1$ was used as the catalyst to assist evaporation of the $\mathrm{B}$ powders. $\mathrm{CH} 4$ and NH3 were used as C and N sources, respectively. The growth of the BCNSs was carried out in a conventional tube furnace. In a typical process $\mathrm{B}$ powders and $\mathrm{CoFe}_{2} \mathrm{O}_{4} / \mathrm{CoO}$ mixture catalyst were mixed and put on the bottom of a crucible, which was then placed at the center of a tube furnace. The mass ratios of the B powders and the mixture catalyst were controlled at 1:4. Subsequently, the reactor temperature was increased to $1300{ }^{\circ} \mathrm{C}$ in $\mathrm{N}_{2}$ at a flow rate of $100 \mathrm{sccm}$. Then $\mathrm{NH}_{3}$ and $\mathrm{CH}_{4}$ were introduced into the reactor with the flow rates of $120 \mathrm{sccm}$ and $10 \mathrm{sccm}$, respectively. The growth time is $1 \mathrm{~h}$. After cooling to room temperature a large amount of gray product could be obtained on the crucible walls. The detailed growth process has also been described in a previous report [32].

The Pt/BCNSs electrocatalysts were prepared using the ethylene glycol (EG) reduction method [33]. 
Firstly, $10 \mathrm{mg}$ BCNSs was put in $10 \mathrm{~mL}$ EG with ultrasonic stirring for $30 \mathrm{~min}$ to obtain a liquid suspension. Then, $0.2 \mathrm{~mL} \mathrm{H}_{2} \mathrm{PtCl}_{6}$ solution in EG (7.5 mg mL-1) was added into the above suspension dropwise with magnetic stirring for $4 \mathrm{~h}$. The $\mathrm{pH}$ value of the suspension was adjusted to above 13 by $\mathrm{NaOH}(2.5 \mathrm{M}$ in EG solution). This mixture was refluxed at $140{ }^{\circ} \mathrm{C}$ for $3 \mathrm{~h}$ to ensure the sufficient reduction of the Pt species. The whole preparation process was protected by an argon flow. Finally, the Pt/BCNSs catalyst with a nominal Pt weight loading of $15 \%$ was obtained after thorough washing with ethanol and drying in vacuum at room temperature.

The structures of the obtained samples were characterized by scanning electron microscopy (SEM, HITACHI S-4700), transmission electron microscopy (TEM, JEM-2100), and X-ray diffraction (XRD, Rigaku $\mathrm{D} / \mathrm{Max} 2500 \mathrm{Pc}$ ). The composition and bonding state of the samples was detected by X-ray photoelectron spectroscopy (XPS, Thermo ESCALAB 250).

The electrochemical performance of the Pt/BCNSs catalyst was tested by an electrochemical workstation (CHI 660C, Shanghai Chenhua Instrument Co., Ltd. China) in $0.1 \mathrm{M} \mathrm{KOH}$ solution. Cyclic voltammogram (CV) and rotating-disk electrode (RDE) polarization curves were measured in a conventional three-electrode electrochemical cell with a platinum wire ( $\mathrm{CHI} 115$ ) as counter electrode and an $\mathrm{Ag} / \mathrm{AgCl}$ (3 $\mathrm{M} \mathrm{KCl}$ solution) (CHI 111) electrode as reference electrode. A glassy carbon (GC) disk (4 mm in diameter, Jiangsu Jiangfen Electroanalytical Instrument Co., Ltd) coated with the Pt/BCNSs catalysts was used as working electrode. Before measurements, the GC disk was washed with distilled water and dried in vacuum. The catalyst suspension was prepared by dispersing $1 \mathrm{mg}$ Pt/BCNSs powders in Nafion solution $(1 \mathrm{~mL}, 0.5 \mathrm{vol} \%$ in ethanol) with sonication for $1 \mathrm{~h}$. Then $10 \mu \mathrm{L} \mathrm{Pt} / \mathrm{BCNSs}$ suspension was dropped onto the GC disk, giving a Pt loading about $1.5 \mu \mathrm{g}$. The RDE polarization curves were measured in the potential range from 0.2 to $-0.8 \mathrm{~V}$ vs $\mathrm{Ag} / \mathrm{AgCl}(3 \mathrm{M} \mathrm{KCl}$ solution) at a scan rate of $10 \mathrm{mV} \mathrm{s}-1$ and rotation rates from 400 to $2500 \mathrm{rpm}$. The CV curves were measured in the same potential range. The current-time (i-t) curves were measured at constant potential of $-0.4 \mathrm{~V}$ for $20000 \mathrm{~s}$ at a fixed rotation rate of $1600 \mathrm{rpm}$.

\section{Results and Discussion}

Fig. 1 shows the SEM images of the BCNSs. It is observed that the BCNSs are spherical and composed of radially oriented nanosheets. The diameters of the BCNSs are in the range of 400-500 nm and the thickness of the constituting nanosheets is measured to be less than $10 \mathrm{~nm}$. It is obvious that this structure can effectively avoid agglomeration of the nanosheets and the spheres.

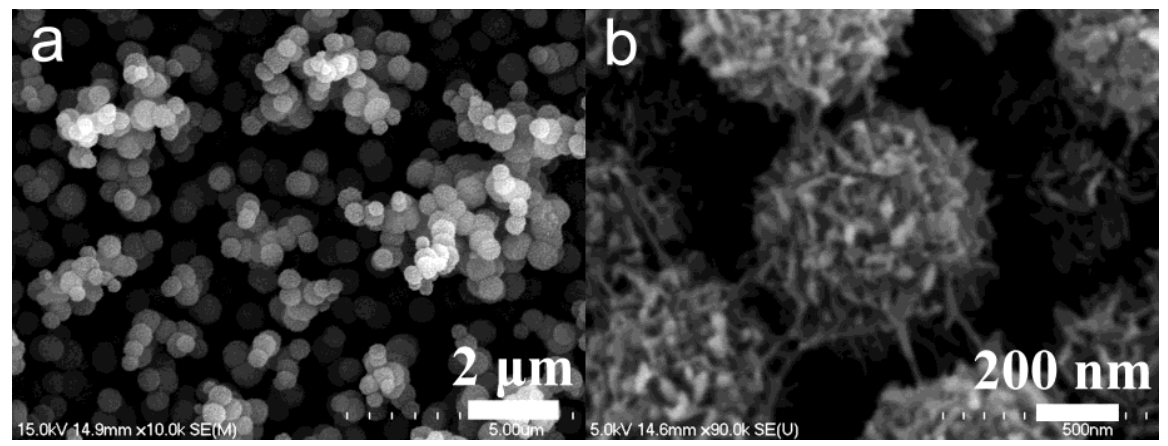

Fig. 1. Magnetization as a function of applied field.

Fig. 2 shows the TEM images of the BCNSs (panels a-c) and Pt/BCNSs catalyst (panels d-f). It is noticed that the BCNSs are hollow and composed of many ultrathin nanosheets, which are radially oriented with the sheet edges exposed on the surface (Fig. 2a and b). These nanosheets are fixed in the BCNS structure and separate from each other, eliminating the problem of agglomeration. The diameter of the spheres is about 
450-500 $\mathrm{nm}$. The constituting nanosheets exhibit a tapered morphology with a mean thickness of $8.6 \mathrm{~nm}$ (Fig. 2a, b). The high resolution TEM (HRTEM) image shown in Fig. 2c clearly reveals straight and parallel lattice fringes in the nanosheets, suggesting the high crystallinity of the BCNSs. The average spacing between adjacent fringes is about $0.33 \mathrm{~nm}$, corresponding to (002) crystal planes of the graphitic BCN [34]. It is considered that the unique structure of the BCNSs is superior for supporting the Pt particles. For example, the BCN nanosheets interconnect and form macropores in the BCNSs. The Pt particles supported on the pore walls can be fully exposed towards the electrolyte. Additionally, the Pt particles supported on the BCNSs subject to less loss during operation because they can still keep contact with the support at a different position even if they separate from the original position on the support. Fig. $2 \mathrm{~d}-\mathrm{f}$ show the TEM images of the Pt/BCNSs catalyst. It is clearly observed that the Pt nanoparticles have successfully been anchored uniformly on the nanosheets of the BCNSs with no agglomeration. The size of the Pt nanoparticles is measured to be in the range of 2-8 $\mathrm{nm}$. This unique BCNSs support, small Pt particle size, and well dispersion of the Pt nanoparticles will lead to high utilization efficiency of the Pt catalyst and excellent electrocatalytic properties.

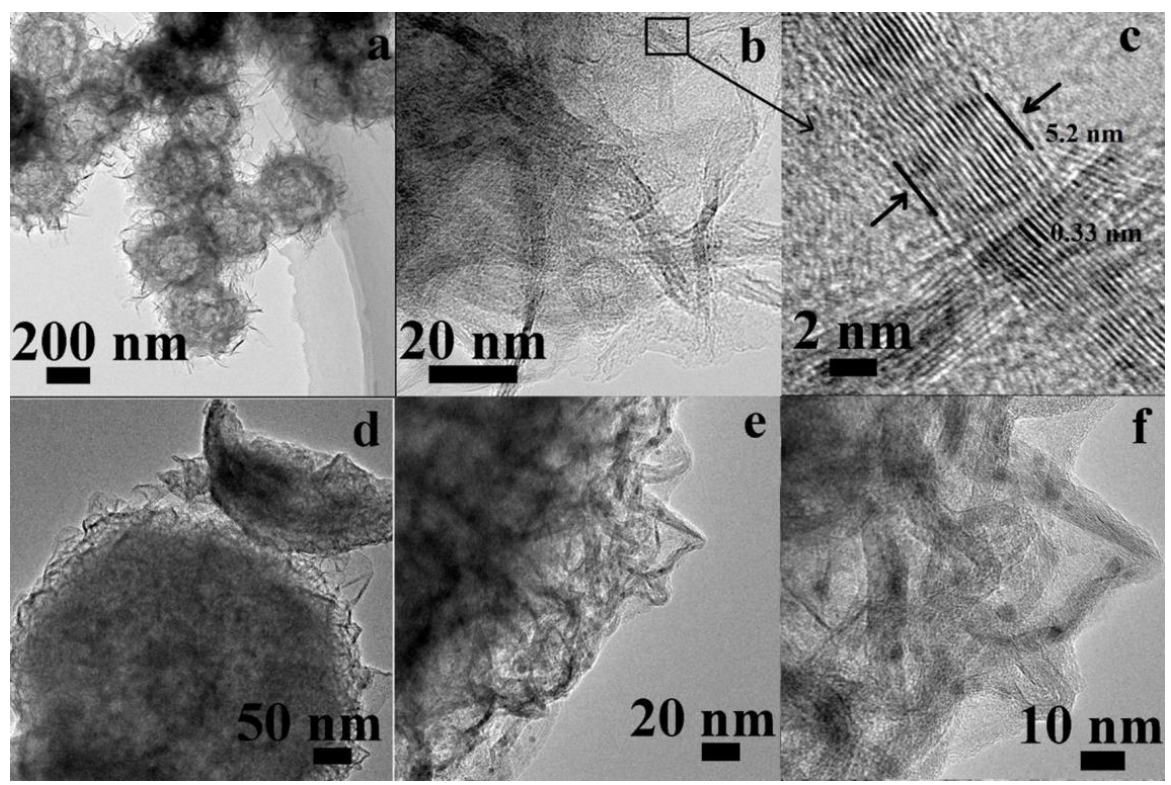

Fig. 2. TEM image of BCNSs (a-c) and Pt/BCNSs catalyst (d-f).

XRD patterns of the BCNSs and Pt/BCNSs are shown in Fig. 3. The pattern of the BCNSs sample exhibits two strong diffraction peaks at $26.6^{\circ}$ and $42.0^{\circ}$, and two weak peaks at $54.8^{\circ}$ and $76.4^{\circ}$, corresponding to the diffractions of (002), (100), (004), and (110) planes of graphitic BCN, respectively [35]. The sharpness of the main peaks indicates that the BCNSs are relatively well crystallized. The Pt/BCNSs catalyst displays several additional diffraction peaks at $39.72^{\circ}, 46.20^{\circ}, 67.52^{\circ}, 81.52^{\circ}$, and $86.14^{\circ}$, which can be ascribed to the diffraction planes of (111), (200), (220), (311), and (222) planes of cubic Pt, respectively (JCPDS, No. 04-0802) [36], [37]. The XRD results suggest that the Pt species were reduced to the metallic state through the EG reduction method.

XPS spectra were measured to determine the chemical composition and bonding states of the BCNSs and Pt/BCNSs samples, as shown in Fig. 4. It can be seen that the BCNSs contain B, C, and N elements while additional Pt and $\mathrm{O}$ elements are present in the Pt/BCNSs sample. The $\mathrm{O}$ component should mainly originate from PtOx species and the unavoidable adsorption of $\mathrm{O} 2$ on the surface. As shown in Figure $4 \mathrm{c}$, the blue lines correspond to the Pt-O bond. Figure $4 \mathrm{~b}$ shows the B 1s, C 1s, and N 1s spectra of the BCNSs. The B 1s peak can be deconvoluted into two peaks at 190.2 and $191.3 \mathrm{eV}$, arising from the B-C and B-N bond, 
respectively [38]. The relatively higher intensity of the B-C peak indicates that more B atoms bond with $\mathrm{C}$ in the network. The three deconvoluted peaks in the $C 1$ s spectrum at 284.4, 284.7, and $285.1 \mathrm{eV}$ could be assigned to C-B, C-C, and C-N, respectively [39]. As can be seen in Figure 4b, the percentage of C-B is higher than that of C-N. N 1s spectrum could be deconvoluted into two separated peaks at 397.8 and 398.4, corresponding to the N-B bond and N-C bond, respectively [40]. The XPS spectra reveal that the BCNSs contain $\mathrm{C}-\mathrm{C}, \mathrm{B}-\mathrm{C}, \mathrm{B}-\mathrm{N}$, and $\mathrm{C}-\mathrm{N}$ bonds, suggesting that the three atoms are atomically bonded, forming a ternary compound. The atomic percentages of $\mathrm{B}, \mathrm{C}$, and $\mathrm{N}$ calculated from the XPS spectra are 15.3, 63.3, 21.4 at.\%, respectively. No additional impurities are present in the samples. Figure 4c shows the $\mathrm{Pt}$ spectrum of the Pt/BCNSs. Pt $4 \mathrm{f}$ peak can be convoluted into two pairs of doublets. The peaks at 71.6 and $75.0 \mathrm{eV}$ (green) can be attributed to Pt $4 \mathrm{f} 7 / 2$ and $4 \mathrm{f} 5 / 2$ of metallic $\operatorname{Pt}(0)$, respectively, and the peaks at 72.75 and $75.35 \mathrm{eV}$ (blue) correspond to Pt(II) [41]. The presence of oxidized Pt species may be attributed to the oxidation of surface Pt in air.

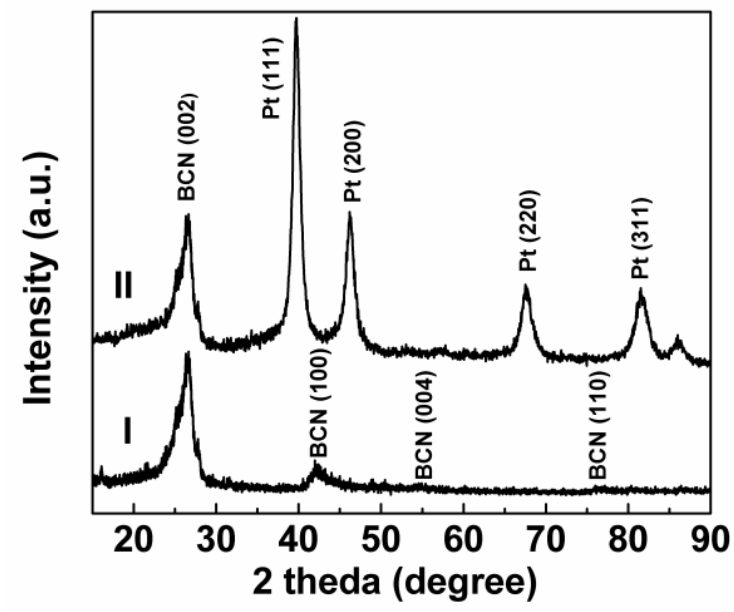

Fig. 3. XRD patterns of BCNSs (a) and Pt/BCNSs catalyst (b).
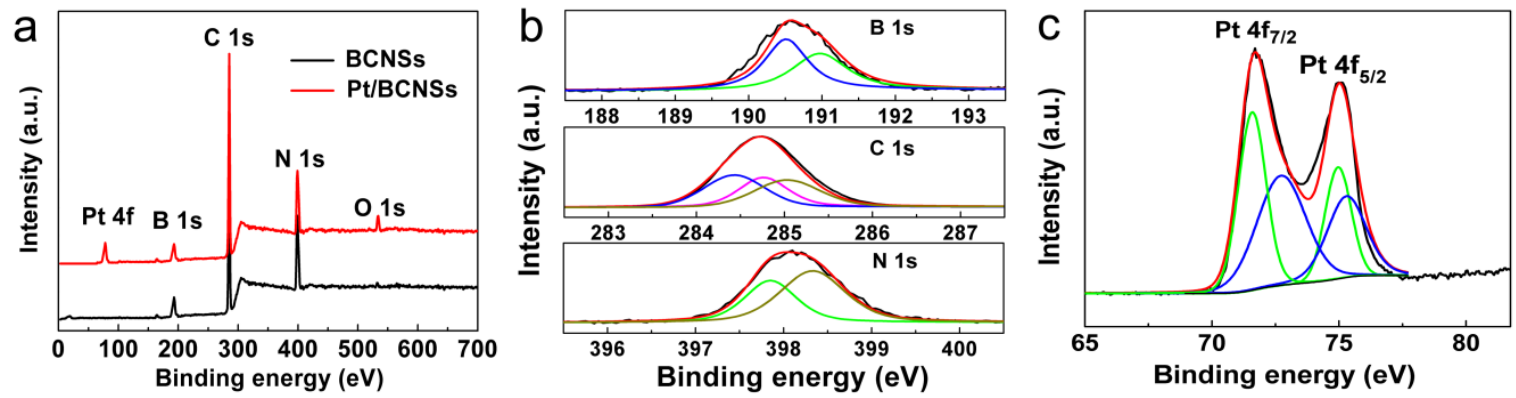

Fig. 4. (a) XPS spectra of BCNSs and Pt/BCNSs catalyst. (b) B 1s, N 1s, and C 1s spectra of BCNSs, in which the blue line corresponds to $\mathrm{B}-\mathrm{C}$ bond, green line corresponds to $\mathrm{B}-\mathrm{N}$ bond, dark yellow line corresponds to C-N bond, and magenta line corresponds to C-C bond. (c) Pt 4f spectrum of the Pt/BCNSs.

The electrocatalytic performance of the Pt/BCNSs catalysts was investigated by measuring the CV and RDE curves in $\mathrm{O}_{2}$-saturated $0.1 \mathrm{M} \mathrm{KOH}$ solution. For comparison, the BCNSs and commercial $20 \% \mathrm{Pt} / \mathrm{C}$ (E-TEK) were also investigated under the same conditions. As shown in Figure 5a, all the CV curves exhibit obvious oxygen reduction peaks, which locate at $-0.35,-0.08$, and $-0.13 \mathrm{~V}$ for BCNSs, Pt/BCNSs, and Pt/C catalysts, respectively. This result indicates that the BCNSs support itself is catalytically active for the ORR. The Pt/BCNSs catalysts exhibit much better electrocatalytic activity for the ORR than the support. Comparing with the $\mathrm{Pt} / \mathrm{C}$ catalyst the $\mathrm{Pt} / \mathrm{BCNSs}$ catalyst displays a more positive peak potential, indicating 
considerable improvement of the catalytic activity for the ORR. The RDE curves were measured to further compare the ORR activity of Pt/BCNSs with that of BCNSs and commercial $20 \% \mathrm{Pt} / \mathrm{C}$ (E-TEK). The RDE curves (Fig. 5b) show a similar changing trend to the CV curves in electrocatalytic activity for the different samples. The onset potentials for BCNSs, Pt/BCNSs, and Pt/C catalysts are $-0.12,0.10$, and $0.05 \mathrm{~V}$ with the corresponding current densities to be $1.08,4.46$, and $5.28 \mathrm{~mA} \mathrm{~cm}^{-2}$ at $-0.40 \mathrm{~V}$, respectively. Obviously, the $\mathrm{Pt} / \mathrm{BCNSs}$ catalyst exhibits more positive onset potential, even better than the $\mathrm{Pt} / \mathrm{C}$ catalyst. This may arise from the synergistic effect mainly between the BCNSs support and Pt.
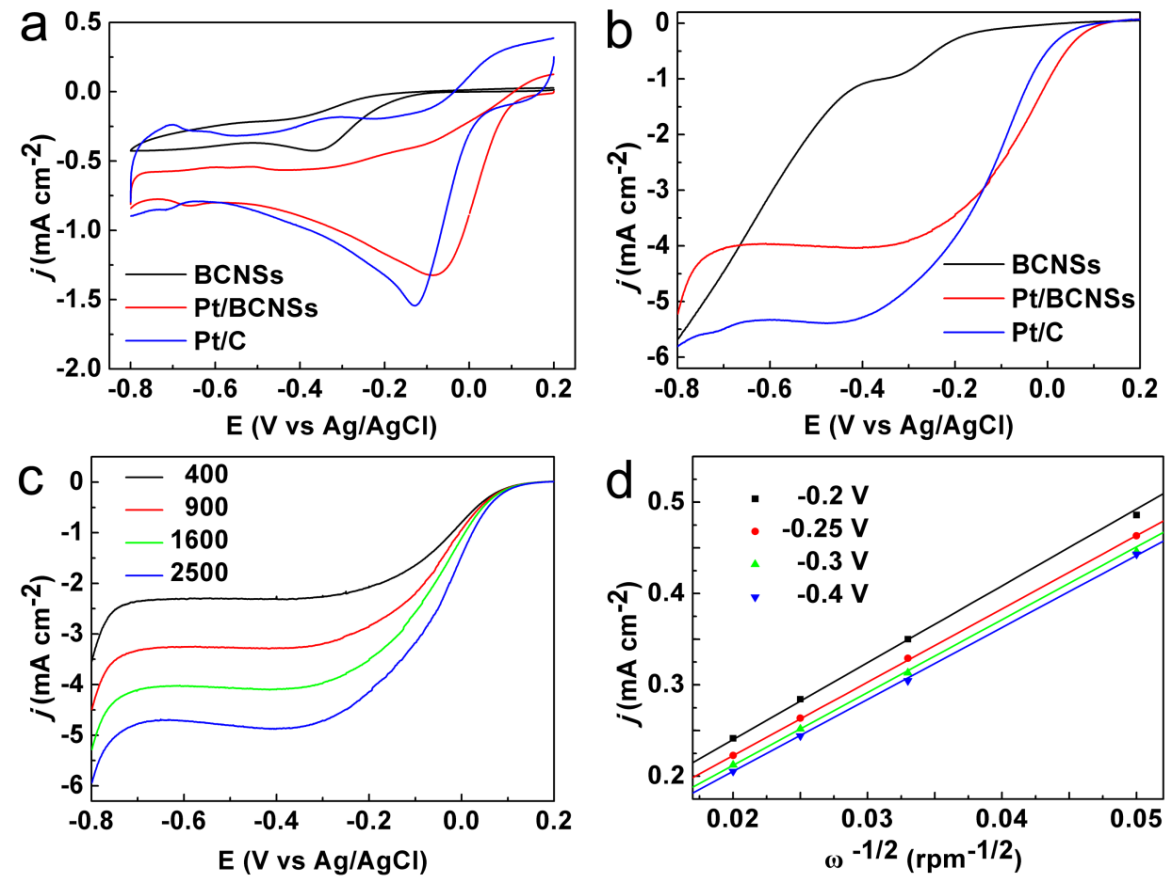

Fig. 5. (a) CV curves of the different samples. (b) RDE curves of the different samples. (c) RDE curves of $\mathrm{Pt} / \mathrm{BCNSs}$ at different rotation speeds. (d) K-L plots calculated from the RDE curves in panel c. (Electrolyte: $\mathrm{O}_{2}$-saturated $0.1 \mathrm{M} \mathrm{KOH}$ solution, scan rate: $10 \mathrm{mV} \mathrm{s}^{-1}$ ).

To further probe into the ORR performance of the Pt/BCNSs catalysts, the RDE curves were tested at different rotation speeds ( 400 to $2500 \mathrm{rpm}$ ). In order to investigate the kinetic process the RDE polarization curves were analyzed using the Koutecky-Levich (K-L) equation described below [42].

$$
\begin{gathered}
\frac{1}{J}=\frac{1}{B \omega^{1 / 2}}+\frac{1}{J_{K}} \\
B=0.62 n F C_{O_{2}} D_{O_{2}}^{2 / 3} v^{-1 / 6} \\
J_{K}=n F k C_{O_{2}}
\end{gathered}
$$

The meaning of the symbols in the equations are as follows: $J$-measured current density, $J_{K}$-kinetic limiting current density, $\omega$-angular velocity of the disk, $n$-overall number of electrons transferred per oxygen molecule during ORR, $D_{\mathrm{O}_{2}}$-diffusion coefficient of $\mathrm{O}_{2}$ molecules in the electrolyte solutions, F-Faraday constant, $\mathrm{C}_{\mathrm{O}_{2}}$-bulk concentration of $\mathrm{O}_{2}$ molecules in the electrolyte solutions, $v$-kinematic 
viscosity of the electrolyte solutions, $k$-electron transfer rate constant. $C_{O_{2}}, D_{O_{2}}$, and $v$ for $O_{2}$-saturated $0.1 \mathrm{M} \mathrm{KOH}$ solution are $1.2 \times 10^{-6} \mathrm{~mol} \mathrm{~cm}^{-3}, 1.9 \times 10^{-5} \mathrm{~cm}^{2} \mathrm{~s}^{-1}$, and $0.01 \mathrm{~cm}^{2} \mathrm{~s}^{-1}$, respectively [42]. Fig. $5 \mathrm{c}$ and d give the RDE curves of the Pt/BCNSs electrode and the corresponding $K-L$ plots, respectively, in which the ORR current densities increased with increasing the rotation speed. In the investigated potential range (0.30-0.45 V), the $K-L$ plots show good linearity and parallelism, indicating that the ORR process over the Pt/BCNSs electrocatalysts follows first-order kinetics with respect to the concentration of oxygen [43]. Calculated from the $K-L$ plots, the average electron number transferred per oxygen molecule is 4.0 . This four-electron transfer process and high kinetic current density confirm the high ORR activity of the Pt/BCNSs electrocatalysts.

The stability of the Pt/BCNSs and commercial $20 \% \mathrm{Pt} / \mathrm{C}$ catalyst was evaluated in alkaline media (Fig. 6). After reaction for $20000 \mathrm{~s}, 85.4 \%$ of the initial current is maintained for the Pt/BCNSs catalyst while the current retention of the commercial Pt/C catalyst is $76.5 \%$. The improved durability of the Pt/BCNSs catalyst is possibly attributed to the excellent corrosion resistance of the BCNSs and less loss as stated above.

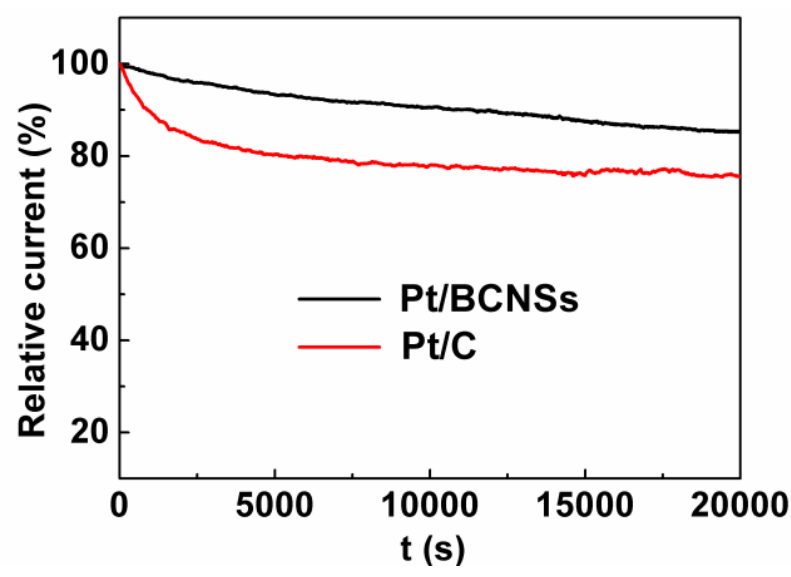

Fig. 6. Current-time (i-t) curves of the Pt/BCNSs and commercial $20 \% \mathrm{Pt} / \mathrm{C}$ (E-TEK) catalyst tested at -0.40 $\mathrm{V}$ in $\mathrm{O}_{2}$-saturated $0.1 \mathrm{M} \mathrm{KOH}$ solution.

\section{Conclusion}

In summary, we have successfully prepared nanosheet-structured BCN spheres (BCNSs) from B powders through a facile catalyzed thermal evaporation approach. The BCNSs are composed of many ultrathin nanosheets, which grow with radial orientation with the sheet edges exposed on the sphere surface. The BCNSs possess excellent performance for application as supports of electrocatalysts for ORR. The prepared Pt/BCNSs catalyst exhibits improved electrocatalytic performance including more positive ORR potential and higher operation stability than the $20 \% \mathrm{Pt} / \mathrm{C}$ catalyst. The unique structure of the BCNSs support and the possible synergistic effect between Pt and the support are responsible for the improvement of the electrocatalytic performance.

\section{Acknowledgment}

This work is supported by the National Natural Science Foundation of China (No. 51272057), and the Shenzhen Basic Research Program (K16295046).

\section{References}

[1] Steele, B. C. H., \& Heinzel, A. (2011). Materials for fuel-cell technologies. Nature, 414, 345-352. 
[2] Li, B., Yan, Z. Y., Higgins, D. C., Yang. D. J., Chen, Z. W., \& Ma, J. X. (2010). Carbon-supported Pt nanowire as novel cathode catalysts for proton exchange membrane fuel cells. J. Power Sources, 262, 488-493.

[3] Li, B., Higgins, D. C., Zhu, S., Li, H., Wang, H., Ma, J., et al. (2012). Highly active Pt-Ru nanowire network catalysts for the methanol oxidation reaction. Catal. Commun., 18, 51-54.

[4] Kim, J. H., Chang, S., Kim, \& Y. T. (2004). Compressive strain as the main origin of enhanced oxygen reduction reaction activity for Pt electrocatalysts on chromium-doped titania support. Appl. Cataly. B: Environ., 158-159, 112-118.

[5] Phuoc, L. T., Huu, C. P., Costa, V. D., \& Janowska, I. (2014). Few-layered graphene-supported palladium as a highly efficient catalyst in oxygen reduction reaction. Chem. Commun., 50, 14433-14435.

[6] Liu, Z. W., Shi, Q. Q., Zhang, R. F., Wang, Q. D., Kang, G J., \& Peng, F. (2014). Phosphorus-doped carbon nanotubes supported low Pt loading catalyst for the oxygen reduction reaction in acidic fuel cells. J. Power Sources, 268, 171-175.

[7] Queiroz, A. C., \& Lima, F. H. B. (2013). Electrocatalytic activity and stability of Co and Mn-based oxides for the oxygen reduction reaction in alkaline electrolyte. J. Electroanal. Chem., 707, 142-150.

[8] Nallathambi, V., Lee, J. W., Kumaraguru S. P., Wu, G., \& Popov, B. N. (2008). Development of high performance carbon composite catalyst for oxygen reduction reaction in PEM proton exchange membrane fuel cells. J. Power Sources, 183, 34-42.

[9] Viva, F. A., Bruno, M. M., Franceschini, E. A., Thomas, Y. R. J., Sanchez, G. R., Feria, O. S., et al. ( 2014). Mesoporous carbon as Pt support for PEM fuel cell. Int. J. Hydrogen Energy, 39, 8821-8826.

[10] Peera, S. G., Tintula, K. K., Sahu, A. K., Shanmugam, S., Sridhar, P., \& Pitchumani, S. (2013). Catalytic activity of Pt anchored onto graphite nanofiber-poly (3, 4-ethylenedioxythiophene) composite toward oxygen reduction reaction in polymer electrolyte fuel cells. Electrochim Acta, 108, 95-103.

[11] Shanmugam, S., \& Momma, T. (2014). Enhanced oxygen reduction activities of Pt supported on nitrogen-doped carbon nanocapsules. Electrochim Acta, 137, 41-48.

[12] Liang, H. W., Zhuang, X. D., Bruller, S., Feng, X. L., \& Mullen, K. (2014). Hierarchically porous carbons with optimized nitrogen doping as highly active electrocatalysts for oxygen reduction. Nat. Commun., 5 , 4973.

[13] Seo, M. H., Choi, S .M., Kim, H. J., \& Kim, W. B. (2011). The graphene-supported Pd and Pt catalysts for highly active oxygen reduction reaction in an alkaline condition. Electrochem. Commun., 13, 182-185.

[14] Zhu, Q., Zhou, S. B. H., Wang, X. Q., \& Dai, S. (2009). Controlled synthesis of mesoporous carbon modified by tungsten carbides as an improved electrocatalyst support for the oxygen reduction reaction. J. Power Sources, 193, 495-500.

[15] Yang, M. H., Wassen, A. R. V., Guarecuco, G., Abruna, H., \& Disalvo, F. J. (2013). Nano-structured ternary niobium titanium nitrides as durable non-carbon supports for oxygen reduction reaction. Chem. Commun., 49, 10853-10855.

[16] Huang, S. Y., Ganesan, P., \& Popov, B. N. (2012). Electrocatalytic activity and stability of titania-supported platinum-palladium electrocatalysts for polymer electrolyte membrane fuel cell. ACS Cata., 2(5), 825-831.

[17] Wang, W., Savadogo, O., \& Ma, Z. (2012). The oxygen reduction reaction on $\mathrm{Pt} / \mathrm{TiO}_{\mathrm{x}} \mathrm{N}_{\mathrm{y}}$-based electrocatalyst for PEM fuel cell applications. J. Appl. Electrochem., 42, 857-866.

[18] Elezovic, N. R., Babic, B. M., Radmilovic, V. R., Vracar, L. M., \& Krstajic, N. V. (2011). Nb-TiO 2 supported platinum nanocatalyst for oxygen reduction reaction in alkaline solutions. Electrochimica Acta, 56(25), 9020-9026.

[19] Liu, H., Wang, F., Zhao, Y., \& Fong, H. (2013). Mechanically resilient electrospun TiC nanofibrous mats surface-decorated with Pt nanoparticles for oxygen reduction reaction with enhanced electrocatalytic 
activities. Nanoscale, 5, 3643-3647.

[20] Liu, Y., \& Mustain, W. E. (2011). Structural and electrochemical studies of Pt clusters supported on high-surface-area tungsten carbide for oxygen reduction. ACS Catal., 1(3), 212-220.

[21] Wu, J. C. S., Fan, Y., \& Lin, C. (2003). Deep oxidation of methanol using a novel Pt/boron nitride catalyst. Ind. Eng. Chem. Res., 42(14), 3225-3229.

[22] Lin, C., Wu, J. C. S., Pan, J., \& Yeh, C. (2012). Characterization of boron-nitride-supported Pt catalysts for the deep oxidation of benzene. J. Catal., 210(1), 39-45.

[23] Wu, J. C. S., Chen, Lin, \& C., S. D. (2005) Boron nitride supported Pt catalyst for selective hydrogenation. Catal. Lett., 102(3-4), 223-227.

[24] Qin, L., Yu, J., Kuang, S. Y., Xiao, C., \& Bai, X. D. (2012). Few-atomic-layered boron carbonitride nanosheets prepared by chemical vapor deposition. Nanoscale, 4, 120-123.

[25] Zhang, T., Zeng, S. F., \& Wen, G. (2014). A simple precursor pyrolysis route to BCN nanoflakes. Mater. Lett., 132, 277-280.

[26] Jin, J. T., Pan, F. P., Jiang, L. H., Fu, X. G., Liang, A. M., Wei, Z. Y., et al. (2014). Catalyst-free synthesis of crumpled boron and nitrogen Co-doped graphite layers with tunable bond structure for oxygen reduction reaction. ACS Nano, 8(4), 3313-3321.

[27] Wang, S. Y., Zhang, L. P., Xia, Z. H., Roy, A., Wook, D., Baek, J. B., et al. (2012). BCN graphene as efficient metal-free electrocatalyst for the oxygen reduction reaction. Angew. Chem. Int. Edit., 51, 4209-4212.

[28] Wang, S. Y., Iyyamperumal, E., Roy, A., Xue, Y. H., Yu, D. S., \& Dai, L. M. (2011). Vertically aligned BCN nanotubes as efficient metal-free electrocatalysts for the oxygen reduction reaction: a synergetic effect by Co-doping with boron and nitrogen. Angew. Chem. Int. Edit., 50, 1-6.

[29] Qiu, Z., Huang, H., Du, J., Tao, X., Xia, Y., Feng, T., et al. (2014). Biotemplated synthesis of bark-structured TiC nanowires as Pt catalyst support with enhanced electrocatalytic activity and durability for methanol oxidation. J. Mater. Chem. A, 2, 8003-8008.

[30] Li, Y., Zhu, E., McLouth, T., Chiu, C., Huang, X., \& Huang, Y. (2012). Stabilization of high-performance oxygen reduction reaction Pt electrocatalyst supported on reduced graphene oxide/carbon black composite. J. Am. Chem. Soc., 134(30), 12326-12329.

[31] Antolini, E. (2012). Graphene as a new carbon support for low-temperature fuel cell catalysts. Appl. Cataly. B: Environ., 123-124, 52-68.

[32] Liu, F., Yu, J., Ji, X. X., \& Qian, M. Q. (2015). Nanosheet-structured boron nitride spheres with a versatile adsorption capacity for water cleaning. ACS Appl. Mater. Inter., 7(3), 1824-1832.

[33] Yue, B., Ma, Y. W., Tao, H. S., Yu, L. S., Jian, G. Q., Wang, X. Z., et al. (2008). CNx nanotubes as catalyst support to immobilize platinum nanoparticles for methanol oxidation. J. Mater. Chem., 18, 1747-1750.

[34] Luo, L. J., Mo, L. B., Tong, Z. F., \& Chen, Y. J. (2009). Facile synthesis of ternary boron carbonitride nanotubes. Nanoscale Res. Lett., 4, 834-838.

[35] Li, X. F., Zhang, J., Shen, L. H., Lei, W. W., Yang, D. P., Cui, Q. L., et al. (2007). Synthesis and characterization of nanocrystalline hexagonal boron carbon-nitride under high temperature and high pressure. J. Phys-Condens. Mat., 19, 425235.

[36] Li, S. S., Zheng, J. N., Wang, A. J., Tao, F. L., Feng, J. J., Chen, J. R., et al. (2014). Branched platinum-on-palladium bimetallic heteronanostructures supported on reduced graphene oxide for highly efficient oxygen reduction reaction. J. Power Sources, 272, 1078-1085.

[37] Kim, Y., Kim, H., \& Kim, W. B. (2014). PtAg nanotubes for electrooxidation of ethylene glycol and glycerol in alkaline media. Electrochem. Commun., 46, 36-39.

[38] Raidongia, K., Nag, A., Hembram, K. P. S. S., Waghmare, U. V., Datta, R., \& Rao, C. N. R. (2010). BCN: A graphene analogue with remarkable adsorptive properties. Chem-Eur. J., 16, 149-157. 
[39] Watannbe, M. O., Itoh, S., Mizushima, K., \& Sasaki, T. (1996). Bonding characterization of $\mathrm{BC}_{2} \mathrm{~N}$ thin films. Appl. Phys. Lett., 68, 2962-2964.

[40] Kim, S. Y., Park, J., Choi, H. C., Ahn, J. P., Hou, J. Q., \& Kang, S. H. (2007). X-ray photoelectron spectroscopy and first principles calculation of BCN nanotubes. J. Am. Chem. Soc., 129, 1705-1716.

[41] Xie, Y. L., Xiao, N., Yu, C., \& Qiu, J. S. (2012). Magnetically recyclable Pt/C(Ni) nanocatalysts with improved selectivity for hydrogenation of O-chloronitrobenzene. Catal. Commun., 28, 69-72.

[42] Wang, C., Daomon, H., Onodera, T., Koda, T., \& Sun, S. (2008). A general approach to the size- and shape-controlled synthesis of platinum nanoparticles and their catalytic reduction of oxygen. Angew. Chem. Int. Edit., 47(19), 3588-3591.

[43] Yin, J., Qiu, Y., \& Yu, J. (2013). Onion-like graphitic nanoshell structured Fe-N/C nanofibers derived from electrospinning for oxygen reduction reaction in acid media. Electrochem. Commun., 31, 1-4.

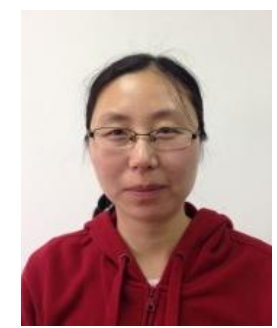

Fei Liu was born in February of 1983 in Jilin of China. She abtained her MS degree from Harbin Engineering University in 2009. Then she joined department of Material Science and Engineering, Shenzhen Graduate School, Harbin Institute of Technology as a graduate and obtained her PhD degree in July, 2015, under the supervision of Prof. Jie Yu. Currently, she is a postdoctoral fellow at southern university of science and technology. Her research interests include the design and synthesis of nanomaterials for catalytic applications and energy storage/conversion.

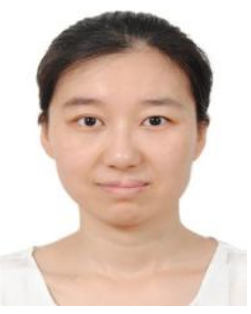

Yaqi Ren was born in June of 1987 in Shanxi of China. She received her BS degree in chemical engineering and technology (2008) and $\mathrm{PhD}$ degree in material science and engineering (2016) from Harbin Institute of Technology, China. Her research focuses on the controlled synthesis of titanium-based compound nanomaterials and their electrochemical properties.

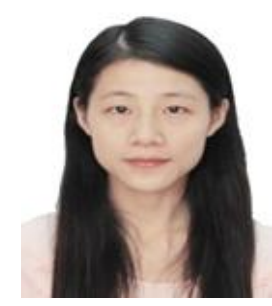

Xixi Ji was born in March of 1990 in Hunan of China. She received her BS degree from School of Metallurgical Engineering, Hunan University of Technology in 2012. Then she received Master's degree from School of materials science and Engineering, Harbin Institute of Technology in 2015, and continued pursuing her $\mathrm{PhD}$ degree at Harbin Institute of Technology under the supervision of Prof. Jie Yu. She is interested in electrospun fibers and diamond, and exploring their application of biosensors. 